\title{
A Rare Case of Intracranial Calcification: Fahr Disease
}

Authors: ${ }^{1}$ Merve Yilmaz, ${ }^{2}$ Asli Tanrivermis Sayit, ${ }^{3}$ Demet Yalcin Kehribar, ${ }^{4}$ Neslihan Unal Akdemir, ${ }^{3}$ Adnan Karatas, ${ }^{3}$ Ozkan Akyol

Hospital:

1Samsun Gazi Governmental Hospital, Endocrinology Department, Samsun, Turkey

2Ondokuz Mayıs University Medical Faculty, Radyology Department, Samsun, Turkey

${ }^{3}$ Samsun Gazi Governmental Hospital, Internal Medicine Department, Samsun, Turkey

${ }^{4}$ Samsun Gazi Governmental Hospital, Neurology Department, Samsun, Turkey

\section{OBJECTIVES}

Introduction: Fahr disease, is a rare pathology characterized by neuropsychiatric symptoms and bilateral symmetrical intracranial, especially basal ganglial calcifications. Although the exact etiology is not known, it is often associated with calcium and phosphorus metabolism disorders. We aim to present two brothers who presented with Fahr 's disease which is a rare condition.

Figure 1: Calcifications in the basal ganglia bilaterally, as well as in the thalamus

Figure 1: Bilateral cerebellar calcification
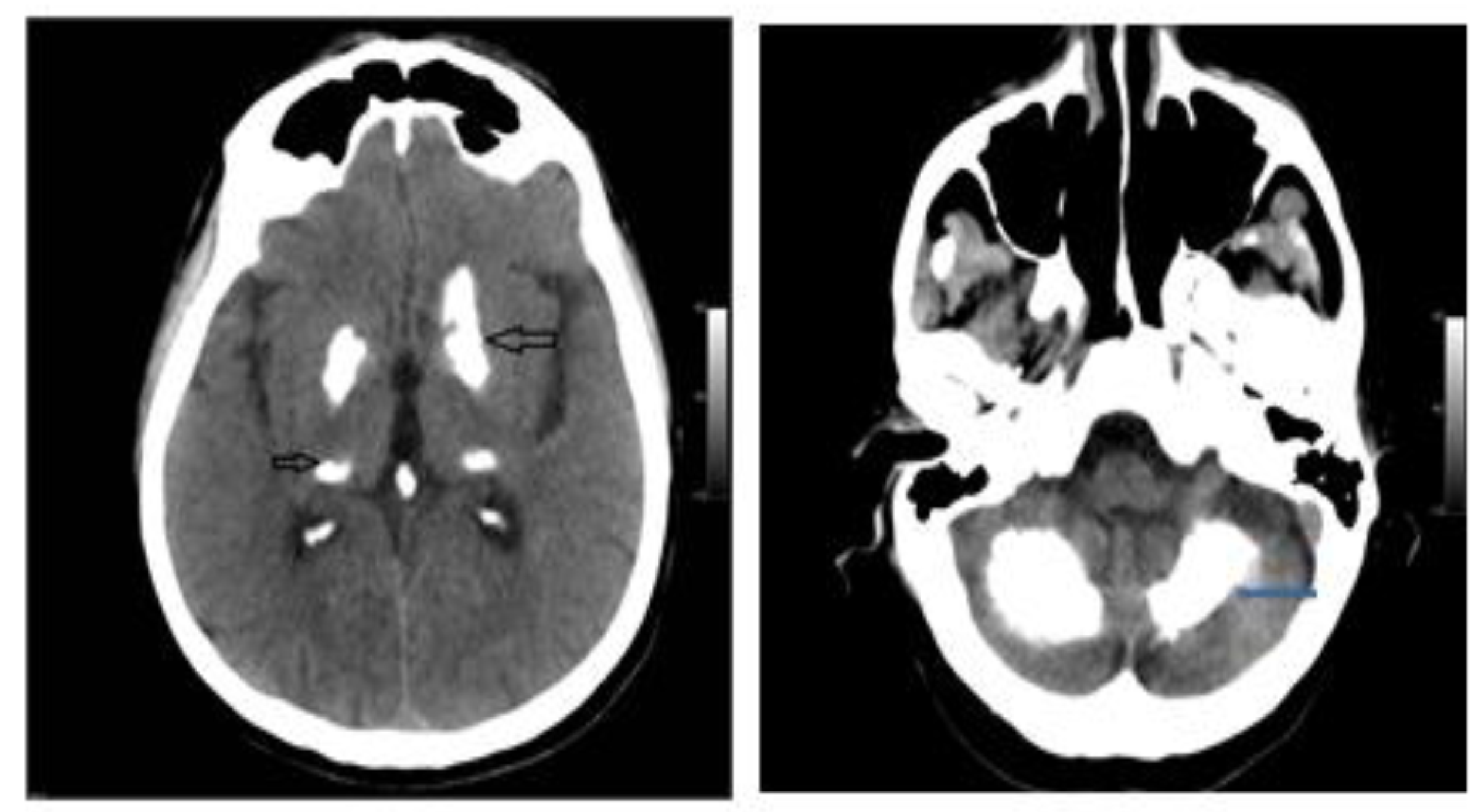

\section{METHODS}

Case: 60 -year-old male patient without a medical history of ilness was admitted to the neurology clinic with speech disorder and seizure. After neurological evaluation of the patient, the brain computerized tomography was performed and revealed bilateral symmetrical calcifications in basal ganglia, thalamus, periventricular white matter, centrum semiovale and dentate nucleus of the cerebellum (Figure1).

Patient was referred to our clinic with the preliminary diagnosis of Fahr disease. In his medical history he had speech disorder for 3 years and received irregular treatment. He didn't have any pathological findings on physical examination. The results of laboratory tests were normal except vitamin $D$ deficiency. The diagnosis of Fahr disease was thought and vitamin $D$ treatment was started. 1 year after brother were evaluated by a neurologist for 5 years with syncope and forgetfulness complaints. Cranial MRI supported Fahr disease. Vitamin D deficiency was found and vitamin $D$ therapy was started. The patients is still being followed by neurology and endocrinology.

\section{RESULTS and CONCLUSIONS}

Patients with Fahr disease frequently admit with neuropsychiatric, extrapyramidal and cerebellar signs, speech impairment and dementia. The association between severity of clinical symptoms and the location and severity of calcification was reported previously. Substitution of calcium and vitamin D corrects the metabolic abnormality and slows down the clinical progression. In conclusion, in patients presenting with neurological symptoms, particularly when bilateral symmetric calcifications of the basal ganglia is detected, the Fahr's disease should be considered in the differential diagnosis and calcium metabolism should be evaluated. 